\title{
Shock breakouts from red supergiants: analytical and numerical predictions
}

\author{
Alexandra Kozyreva ${ }^{1 \star}$, Ehud Nakar ${ }^{2}$, Roni Waldman ${ }^{3}$, \\ Sergei Blinnikov ${ }^{4,5,6}$, Petr Baklanov ${ }^{4,5,7}$ \\ ${ }^{1}$ Max-Planck-Institut für Astrophysik, Garching bei München, 85748, Germany \\ 2 The Sackler School of Physics and Astronomy, Tel Aviv University, Tel Aviv, 6997212, Israel \\ ${ }^{3}$ Racah Institute of Physics, The Hebrew University, Jerusalem 91904, Israel \\ ${ }^{4}$ Space Research Institute (IKI), 84/32 Profsoyuznaya, Moscow, 117997, Russia \\ ${ }^{5}$ NRC "Kurchatov Institute" - ITEP, Moscow, 117218, Russia \\ ${ }^{6}$ Kavli IPMU (WPI), University of Tokyo, Kashiwa, Chiba 277-8583, Japan \\ ${ }^{7}$ National Research Nuclear University Moscow Engineering Physics Institute, Moscow, 115409, Russia
}

Accepted XXX. Received YYY; in original form ZZZ

\begin{abstract}
Shock breakout (SBO) signal is the first signature of the supernova explosion apart from gravitational waves and neutrinos. Observational properties of SBO, such as bolometric luminosity and colour temperature, connect to the supernova progenitor and explosion parameters. Detecting SBO or SBO-cooling will constrain the progenitor and explosion models of collapsing stars. In the light of recently launched eROSITA telescope, the rate for detection of SBO is a few events during a year. In the current study, we examine the analytic formulae derived by Shussman et al. (2016). We use four red supergiant models from their study, while running explosions with the radiation hydrodynamics code STELLA. We conclude that there is a good agreement between analytic and numerical approaches for bolometric luminosity and colour temperature during SBO. The analytic formulae for the SBO signal based on the global supernova parameters can be used instead of running time-consuming numerical simulations. We define spectral range where analytic formulae for the SBO spectra are valid. We provide improved analytical expression for the SBO spectral energy distribution. We confirm dependence of colour temperature on radius derived by analytical studies and suggest to use early time observations to confine the progenitor radius. Additionally we show the prediction for the SBO signal from red supergiants as seen by eROSITA instrument.
\end{abstract}

Key words: supernovae: general - supernovae - stars: massive - radiative transfer

\section{INTRODUCTION}

Nowadays, it is become possible to detect supernovae at very early phase and resolve the light curve behavior very soon after the supernova explosion. With short-cadence surveys observers are able to catch the earliest light indicating the explosion Arcavi et al. (2017). The first electro-magnetic signature of the explosion of a massive star is the shock breakout (SBO) event, i.e. the emergence of the shock on the surface of the progenitor star. However, the SBO peaks in extreme ultraviolet, while the majority of surveys specified in longer wavelengths. So far, there are only few SBO events detected (Gezari et al. 2008; Schawinski et al. 2008;

* E-mail: sasha@mpa-garching.mpg.de
Soderberg et al. 2008; Modjaz et al. 2009). The difficulty consists in the short duration of the SBO, spanning minutes for compact progenitors (fraction of solar radius to hundred solar radii) to hours for extended progenitors, e.g. red supergiants (hundreds to thousands solar radii).

One of the most important aspects of the SBO is that the SBO properties like bolometric luminosity and temperature depend on the progenitor radius and indirectly depend on ejecta mass and explosion energy. Hence, detecting SBOs from normal core-collapse supernovae (CCSNe) which originate from the explosion of red supergiants (Smartt 2009) will help to constrain progenitor and explosion properties. Estimates for the unknown characteristics like progenitor radius and explosion energy from the SBO phase serve as an additional constrain to the first-order approximations for 
supernova parameters from Litvinova \& Nadezhin (1985); Popov (1993) and latest corrected relationships Goldberg et al. (2019) and will break the parameter degeneracy of the explosion models (Dessart \& Hillier 2019). One of the possible ways to derive the required parameters is to compare the SBO properties to the analytic formulae or numerical simulations. While detailed numerical simulations lead to a more exact model, analytical approach provides the simplest procedure to get approximate values for the progenitor.

Amongst others, Nakar \& Sari (2010), Rabinak \& Waxman (2011), and later Shussman et al. (2016) derive the analytic prescription for the early light curve of SBO, the color temperature, and spectra. Shussman et al. (2016) calibrate the analytic relations with the numerical simulations based on 120 red supergiant models. While solving the reverse problem using the analytic formulae, one may estimate progenitor and explosion parameters from the observed light curve, temperature, and spectra without running timeconsuming numerical simulations of stellar evolution and explosion models.

In the present study, we run a few models from the Shussman et al. (2016) set using more sophisticated multigroup radiation hydrodynamics code (STELLA) and confirm reasonable agreement to their analytic prescription. We describe the models and the method in Section 2, compare our numerical results with the analytic formulae in Section 3, analyse the spectral range in which analytic expressions provide reliable fits, and summarise the current study in Section 5, while offering the improved relations for the SBO signature.

\section{MODELS AND METHOD}

For our study we selected four core-collapse progenitor models from Shussman et al. (2016). Among 120 progenitors from their study, we chose three typical progenitors that cover almost the entire range of radii $\left(345-1024 M_{\odot}\right)$ and one progenitor that is extreme, with relatively smaller radius and atypical density structure. These are: m12115rot2, m1215rot2, m15115rot0, m1515rot8 - all computed with MESA $^{1}$. The Table 1 contains some characteristics of our models such as radius, final and ejecta mass, parameters used for stellar evolution calculations, such as a parameter of Ledoux convection and rotation in units of Keplerian equatorial velocity. All stellar models are at solar metallicity, calculated with semiconvection parameter 0.1 and exponential overshoot formula with the parameter of 0.008 . In Figure 1, we show the density structure of all four models. The radius varies in a wide range from $268 R_{\odot}$ to $1024 R_{\odot}$, while density gradient stays the same for all models except the model m1515rot8, which has a sharper slope of density in the hydrogen-rich envelope.

For the explosion and post-explosion evolution we used the radiation hydrodynamics code STELLA (Blinnikov et al. 2006). The explosion is initiated in STELLA as a thermal bomb. 1.1 foe of thermal energy ( 1 foe $\equiv 10^{51} \mathrm{erg}$ ) is injected at the bottom of the inner boundary in the inner

1 Modules for Experiments in Stellar Astrophysics http://mesa. sourceforge.net/ (Paxton et al. 2011, 2013, 2015, 2018).
Table 1. Characteristics of the red supergiants used in the present study. Radius is in solar radii. Final and ejecta masses are in solar masses. "Convection" stands for the parameter of Ledoux convection. "Rotation" means equatorial velocity in units of Keplerian velocity.

\begin{tabular}{lrcllc}
$\begin{array}{l}\text { model } \\
\text { name }\end{array}$ & $\begin{array}{r}\text { Radius } \\
{\left[R_{\odot}\right]}\end{array}$ & $\begin{array}{c}M_{\text {final }} \\
{\left[M_{\odot}\right]}\end{array}$ & $\begin{array}{l}M_{e j} \\
{\left[M_{\odot}\right]}\end{array}$ & Convection & Rotation \\
\hline m12115rot2 & 812 & 11.09 & 8.09 & 1.5 & 0.2 \\
m1215rot2 & 345 & 11.17 & 9.15 & 5 & 0.2 \\
m15115rot0 & 1024 & 13.33 & 11.33 & 1.5 & - \\
m1515rot8 & 268 & 8.3 & 6.1 & 5 & 0.8
\end{tabular}

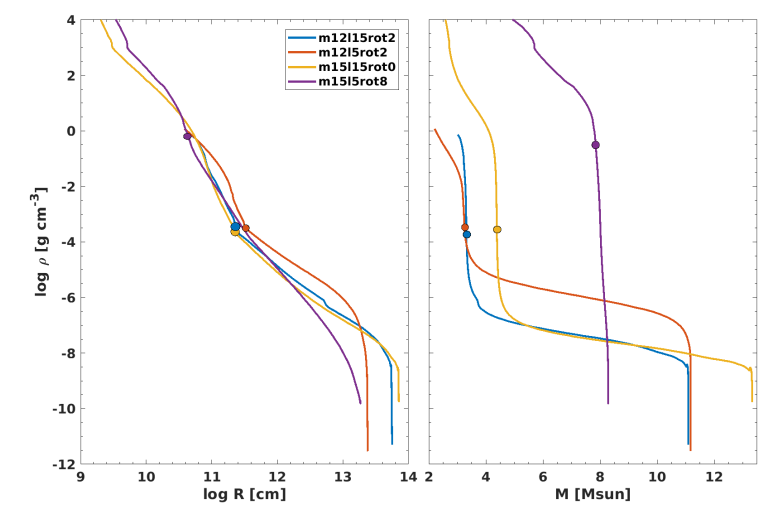

Figure 1. Density structure of the red supergiant models in the study. Dots on each curve indicate the bottom of hydrogen-rich envelope.

$0.3 M_{\odot}$ of the supernova ejecta, on top of the region which is considered to collapse into a proto-neutron star. By the end of simulations (1-2 days after the explosion), the final kinetic energy is about 1 foe for all models. In the model m12115rot2 we truncated the inner $4 M_{\odot}$ to simplify calculations to avoid some computational issues. Nevertheless the exact inner structure does not affect the resulting shock breakout properties and does not influence the conclusions of the present study. In general, the simulations are carried out with the standard opacity treatment, and with the standard numerical parameter set used for the majority of STELLA studies. We pay special attention to the parameter BQ which is designed to resolve SBO evolution according to Tominaga et al. (2011). The standard value is $B Q=1$, while we set $\mathrm{BQ}=0$ which is needed specifically for the SBO simulations. The parameter is responsible for the efficiency of energy conversion from kinetic energy of the shock into thermal energy (see detailed explanation in Section 2.5.2.3 Moriya 2013). The value $\mathrm{BQ}=0$ represents the idealised $100 \%$-efficiency of energy conversion and corresponds to Rankine-Hugoniot conditions. On top of that, BQ "smears" velocity and density gradients among a few meshes (see Appendix in Blinnikov et al. 1998) to mimic multi-dimensional effects which develop during the ejecta expansion (Badjin et al. 2016). The ejecta is spherical and "compact" at the moment of SBO relative to the later supernova phases. Later different multidimensional instabilities appear, and this makes conversion 


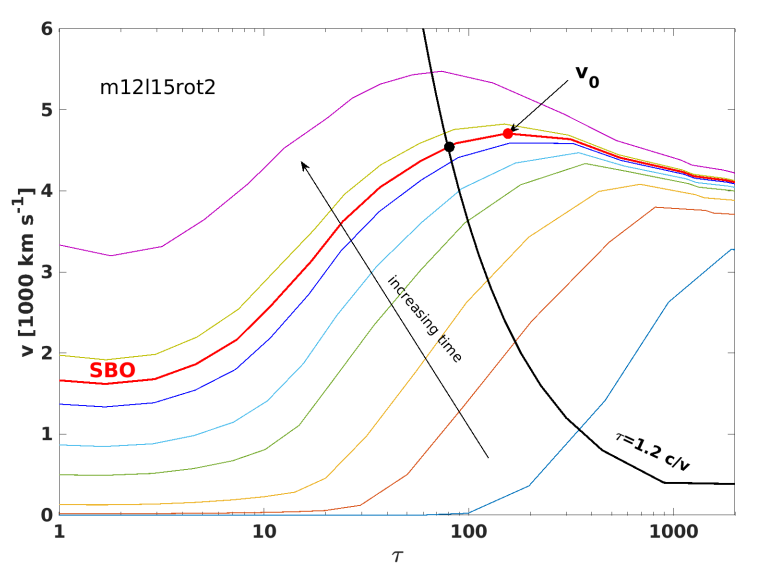

Figure 2. The choice for $v_{0}, \rho_{0}$ of the breakout shell required for calculating SBO properties.

of kinetic energy less efficient. Keep running simulations for normal supernovae with $\mathrm{BQ}=0$ on the longer time scale provides less physical results (see also Fig. 4.13, Section 4.3.3.2 in Moriya 2013).

The spectral energy distribution (SED) is computed in the wavelength spanning from $1 \AA$ to $50,000 \AA$. The frequency range is divided on 100 bins logarithmically, in which the radiative transfer equations are solved at every time step. The final bolometric light curve is integrated over the spectra. Colour temperature is estimated as a black body temperature via the least-square method.

We emphasize that in the present analysis we do multigroup radiation simulations coupled with hydrodynamical evolution of the ejecta which is the next step in the complexity after gray-opacity calculations done by Shussman et al. (2016). Of course, there are a number of published SBO studies, e.g. Ensman \& Burrows (1992, onetemperature simulations), Tominaga et al. (2011, STELLA) and Tolstov et al. (2013, STELLA), Morozova et al. (2016, gray-opacity, diffusion approximation, SNEC), Lovegrove et al. (2017, gray-opacity, CASTRO), Dessart et al. (2017, HERACLES/non-LTE CMFGEN) and others.

\section{COMPARISON BETWEEN NUMERICAL AND ANALYTICAL APPROACH}

\subsection{Bolometric light curves and color temperature}

We compare the resulting light curves for the models described in Section 2 with those calculated with analytic formulae from Shussman et al. (2016). In particular, we used their formulae (33) and (34), (39) and $(41)^{2}$ which define SBO bolometric luminosity and colour temperature from either (1) SBO parameters, i.e. breakout-shell velocity $v_{0}$ and density ahead the shock $\rho_{0}$ (Eq. 33 and 34), or (2) global

2 These formulae are implemented in the web interface http: //www.astro.tau.ac.il/ tomersh/. We list some formulae from Shussman et al. (2016) which we explore in the current study in Appendix A for convenience. supernova parameters, i.e. ejecta mass, radius of the progenitor and the explosion energy (Eq. 39 and 41, which are implemented on the mentioned website). The $v_{0}$ is defined as the maximal shock velocity when the outermost mass zone reaches $1 / 3$ of the maximal velocity which corresponds to the moment when outcoming luminosity equals the peak luminosity of the SBO. As discussed by Shussman et al. 2016 (see their Section 3.1), $v_{0}$ and $\rho_{0}$ should be picked from the condition $\tau=1.2 c / v$ instead of $\tau=c / v_{0}$, where $\tau$ is Rosseland mean optical depth. We demonstrate the procedure of the choice in Figure 2. Here, the red curve corresponds to the profile when the peak luminosity is reached and when velocity at the surface of the progenitor is $1 / 3$ of the shock velocity (i.e. maximal velocity). Figures 3 and 4 present the comparison plots. Note that the $\mathrm{X}$-axis in all the plots in the paper is in logarithmic scale, therefore, the first point of each curve is at time $=0$ relative to the maximum SBO luminosity and could not be plotted. By default, luminosity and temperature are constant before the curves begin on the plots, i.e. before $100 \mathrm{~s}$. We find out that in both cases peak bolometric luminosity (horizontal part of the dashed and blue curves) is overestimated by a factor of at least $0.5 \mathrm{dex}$ if calculated via Eq. 33 using the values of breakout-shell parameters $v_{0}$ and $\rho_{0}$ and compared to numerically simulated. Nevertheless, the "analytical" peak luminosity is very sensitive to the value of breakout shell velocity (to the exponent of 3) and density (to the exponent of 1). Note also, that density just on front of the breakout shell changes dramatically, and the choice of density value $\rho_{0}$ strongly affects the resulting peak luminosity, therefore, it is a source of large uncertainty. On top of that, calibration done by Shussman et al. (2016) was carried out with the simplified gray-opacity radiation code, which provides a first-order estimate of the evolution of radiation field and hydrodynamical evolution tightly coupled with radiation. Comparison shows that analytical estimate based on breakout shell parameters for the peak luminosity deviates from numerical simulations to $0.5-1$ dex. Apart from that, in Figure 3 and 4, we present analytical curves calculated from equation (39) from Shussman et al. (2016) using global progenitor and supernova parameters (black solid curves), i.e. ejecta mass, radius of the progenitor and the explosion energy, and which are available via website http://www. astro.tau.ac.il/ tomersh/. These analytical curves suit better the numerical curves deviating from the numerical approach to 0.2 dex, i.e. reaching agreement within a factor of 3. Maximum breakout luminosity is different if compare the present numerical simulations and calibrated analytical values: $\log L_{b o l}^{\max }=45.2 \mathrm{erg} \mathrm{s}^{-1}, 45.0 \mathrm{erg} \mathrm{s}^{-1}, 45.0 \mathrm{erg} \mathrm{s}^{-1}$, and $44.9 \mathrm{erg} \mathrm{s}^{-1}$ in STELLA simulations, while $\log L_{b o l}^{\max }=$ $45.4 \mathrm{erg} \mathrm{s}^{-1}, 45.4 \mathrm{erg} \mathrm{s}^{-1}, 45.3 \mathrm{erg} \mathrm{s}^{-1}$, and $45.6 \mathrm{erg} \mathrm{s}^{-1}$ from Eq. 39 (Shussman et al. 2016) for the model m12115rot2, m1215rot2, m15115rot0, and m1515rot8, correspondingly. Hence, Shussman et al. (2016) calibration overestimates the peak luminosity what leads to incorrect evaluation of energetics during the maximum light.

We derive a broken-power law for the bolometric light curves and colour temperature evolution for our present simulations. We summarise the values of the average exponents in Table 2. For this, each STELLA bolometric luminosity curve is divided on 3 linear pieces in logarithmic scale in accordance with convenient physical conditions. These are maximum breakout luminosity phase, planar phase, and 
so-called spherical phase. Each piece is approximated by $\log L_{b o l}=a+b \log t$, where $t$ is time in seconds since SBO. In Table 2, we also present slopes in the luminosity and colour temperature evolution published in other analytical studes for comparison.

Colour temperature is analysed similar to bolometric luminosity, i.e. $\log T_{c o l}=c+d \log t$. Maximal temperature $\log T_{\text {col }}^{\max }$ is in K: 5.5, 5.6, 5.4 and 5.7 in STELLA, and 5.6, 5.8, 5.5, and 5.9 from Eq. 41. We conclude that temporal evolution of SBO luminosity and colour temperature based on the detailed numerical simulations with STELLA agree well with corresponding evolution presented in Shussman et al. (2016) while peak values are overestimated in Shussman et al. (2016) in comparison to STELLA.

To conclude, correct estimate of the colour temperature provides qualitatively adequate model for spectral maximum and the integrated flux over the spectral maximum. This is very important for the prediction of progenitor parameters from earlier observations of supernovae.

\subsection{Effect of light travel time}

A special attention was paid by Shussman et al. (2016) to the effect of light travel time (see their Section 4.3). This is a geometrical effect which takes into consideration that photons emitted at an angle $\theta$ to the line of sight require longer time to travel to the observer. In the extreme case of $\theta=90^{\circ}$, the delay is $R / c$, where $R$ is the photon emission radius. This leads to smearing of the light curve over a duration of $R / c$. STELLA does not account for this effect, consequently, the numerical results are in reasonable agreement with analytical formulae with no effect of light travel time included. Since the breakout velocity is much lower than the speed of light, the light travel time affects the light curve significantly only during the initial light crossing time of the progenitor, namely $t \lesssim R_{\text {prog }} / c$. If one is interested in the light curve during this time then we recommend that the light travel time would be included. We found out that there is a missing factor of $\cos (\theta)$ in the integrand on the right-hand side of Eq. 22 of Shussman et al. (2016, Section 4.3). As a result the entire treatment of the light travel time in Shussman et al. (2016) is mistaken (including the factor $f$ they finds). A good approximation of the light travel time effect is obtained by the following formula:

$L_{l t t}(t)=\frac{2}{t_{R c}} \int_{t-t_{R c}}^{t} L\left(t^{\prime}\right)\left(1-\frac{\left(t-t^{\prime}\right)}{t_{R c}}\right) d t^{\prime}$,

where $t_{R c}=R_{\text {prog }} / c$.

For a more accurate treatment see Katz et al. (2012). Note that for $t<t_{R c}$ the integrand on the right-hand side includes also contribution from $t^{\prime}<0$ (i.e., before the peak of $L(t))$. This contribution must be taken into account. An analytic approximation of $L(t<0)$ is given by Eq. 13 of Shussman et al. (2016).

\subsection{Spectral Energy Distribution}

According to Eq. 19 and 21 from Shussman et al. (2016), the spectral energy distribution (hereafter, SED) of SBO pulse at given time is:

$L_{\nu}=0.9 L \cdot \frac{15}{\pi^{4}}\left(\frac{h}{3 k T_{\mathrm{col}}(\nu)}\right)^{4} \nu^{3} \cdot\left(e^{h \nu / k T_{\mathrm{col}}(\nu)}-1\right)^{-1}$

where

$T_{\mathrm{col}}(\nu) \approx T_{\mathrm{obs}}\left(\frac{h \nu}{3 k T_{\mathrm{obs}}}\right)^{0.2}$,

where $k=1.38 \times 10^{-16} \mathrm{erg} \mathrm{K}^{-1}$ is Boltzmann constant, $h=6.626 \times 10^{-27} \mathrm{erg} \mathrm{Hz}^{-1}$ is Planck constant. $T_{\text {obs }}$ and $L=L_{\text {obs }}$ are generally calculated via formulae (33) and (35) or (39) and (41) from Shussman et al. (2016). For demonstration, we apply $T_{\mathrm{obs}}=T_{\mathrm{col}}^{\mathrm{STELLA}}$ and $L=L_{\mathrm{bol}}^{\text {STELLA }}$.

In Figure 5, the SED for model m12115rot2 are taken at $300 \mathrm{~s}$ after the SBO pulse. These are: SED from the STELLA numerical simulations (red curve) and analytical spectra (blue curve). The maxima perfectly coincide, i.e. the colour temperature is estimated correctly in analytic prescription at this epoch. Additionally we show the black-body SED at emitting radius $R_{B B}$ (so-called, black-body radius) taken from the STELLA results. Pure black-body underestimates number of low-energy photons because of dilution in the semi-transparent ejecta (Sobolev 1985). Rayleign - Jeans approximation is shown as green dashed curve and taken at radius where Rosseland optical depth equals $2 / 3$. In fact, spectrum at a given moment is the sum of black body spectra at different colour temperatures, i.e. the observer sees different layers at different frequencies, since optical depth is frequency dependent. Hence, the resulting spectrum deviates from a pure black body SED (Blinnikov \& Tolstov 2011). The plot shows fairly good agreement between the analytic prescription Eq. 2 (Eq. 21, Shussman et al. 2016) and numerical simulations done with STELLA around spectral maximum, as expected. However, we find out that there is a systematic offset of about 0.1 dex between numerical and analytic curves most likely resulting from the factor 0.9 in Eq. 2 in Shussman et al. (Eq. 21 2016).

Among other features, there is a cut-off in numerically computed spectrum at higher frequencies due to photoionisation (bound-free transitions) and line-blanketing while there is no cut-off in analytically calculated spectrum because the assumptions do not include influence of photoionisation and effect of line opacity.

In Figure 6, we show the upper and lower frequencies which limit the feasibility range of the given analytical expressions. We define the limiting frequencies where the difference between analytic SED deviates from numerical SED less than $1 \%$ (in log-scale), i.e. where

$\frac{\log F_{\nu}^{\mathrm{an}}(\nu)-\log F_{\nu}^{\mathrm{num}}(\nu)}{\log F_{\nu}^{\mathrm{num}}(\nu)}<0.01$.

In Figure 6, the frequency limits are shown for the quantity $h \nu / k T_{\text {col }}$. The noisy behaviour of the curves is unavoidable and caused by the numerical nature of the data. Thus, the upper and lower limits for analytic SED follow colour temperature evolution, so that analytical formulation of Shussman et al. (2016) is valid in the range of about $10 \times\left(\frac{h \nu}{k T_{\mathrm{col}}}\right)$ around spectral maximum. Major flux is radiated between these limiting frequencies, while there is no significant contribution at higher frequencies due to line-blanketing and Compton scattering, and there is a little contribution at 
Table 2. Broken-power law fits for $L_{b o l}$ and $T_{c o l}$ derived from the present numerical simulations, and the exponents from analytical formulae from Nakar \& Sari (2010), Rabinak \& Waxman (2011), Sapir et al. (2011), Shussman et al. (2016), Sapir \& Waxman (2017), and Faran et al. (2019).

\begin{tabular}{lcccc}
\hline Source & \multicolumn{2}{c}{ Lbol } & \multicolumn{2}{c}{ Tcol } \\
& $\begin{array}{c}\text { Planar } \\
\text { phase }\end{array}$ & $\begin{array}{c}\text { Spherical } \\
\text { phase }\end{array}$ & $\begin{array}{c}\text { Planar } \\
\text { phase }\end{array}$ & $\begin{array}{c}\text { Spherical } \\
\text { phase }\end{array}$ \\
\hline Rabinak \& Waxman (2011) & & $0.35 / 0.16$ & & $0.45 / 0.47$ \\
Sapir et al. (2011) & $4 / 3$ & & 0.2 & 0.5 \\
Sapir \& Waxman (2017) & & & 0.27 & $0.86 / 0.41 / 0.6 / 0.56$ \\
Faran et al. (2019) & $4 / 3$ & $2 / 30.36$ & 0.56 \\
Nakar \& Sari (2010) & $4 / 3$ & 0.17 & 0.36 & 0.6 \\
Shussman et al. (2016) & $4 / 3$ & 0.35 & $0.45 / 0.55$ & 0.54 \\
\hline STELLA & 1.22 & 0.35 & $0.48 / 0.27$ & 0.5
\end{tabular}
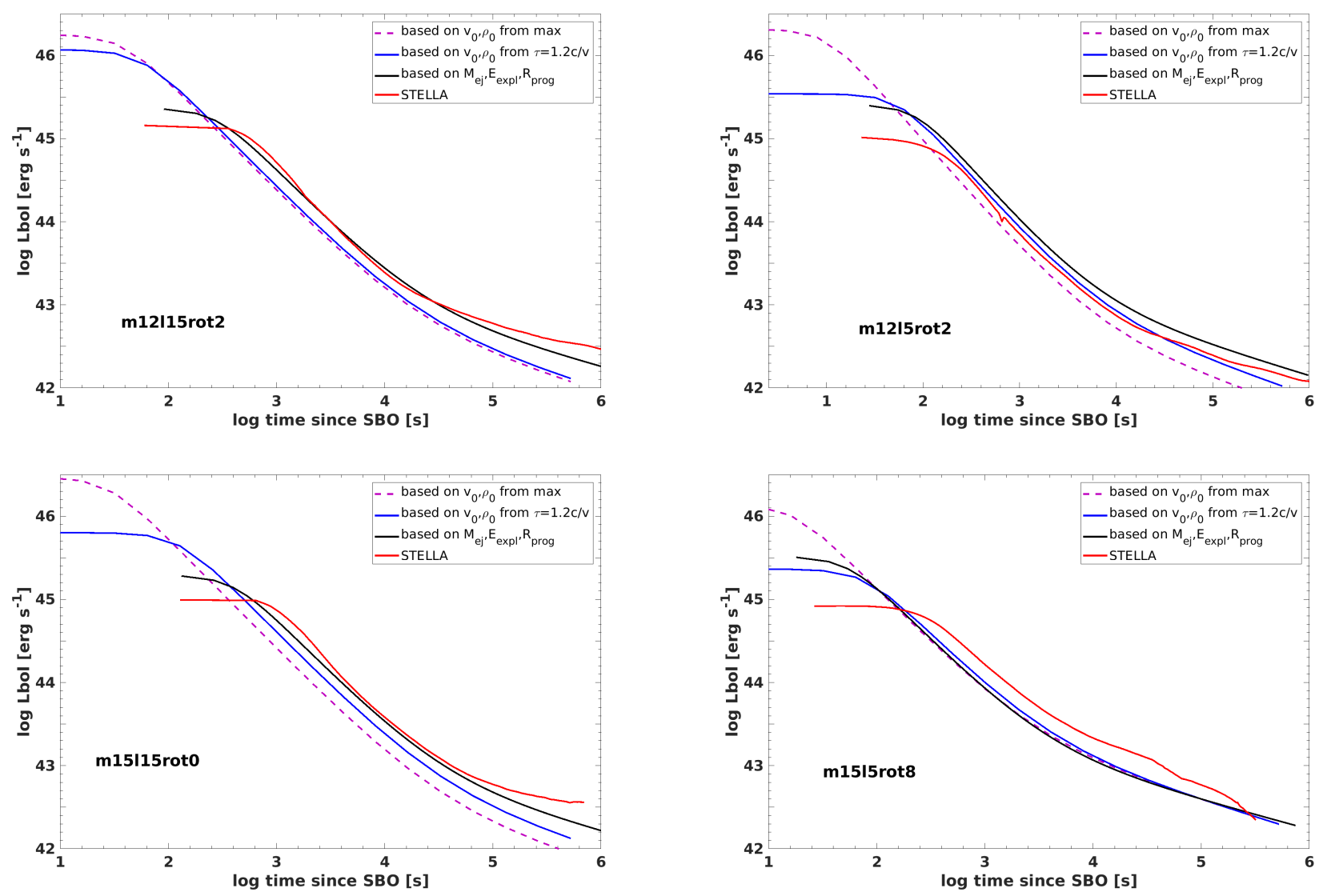

Figure 3. SBO bolometric light curves for red supergiant models m12115rot2, m1215rot2, m15115rot0, and m1515rot8. The dashed curve represents bolometric luminosity from Eq. 33 (see Appendix A) based on breakout shell parameters $v_{0}, \rho_{0}$ at maximum shock velocity. The blue curve is calculated with the same Eq. 33 based on $v_{0}, \rho_{0}$ at the point where $\tau=1.2 c / v_{0}$. The black curve is calculated with Eq. 39 based on global supernova properties $M_{e j}, E_{\text {expl }}, R_{\text {prog. }}$. See discussion in the text.

lower frequencies, which can be estimated from RayleighJeans formula. In other words, analytical formulae by Shussman et al. (2016) are valid in the following wavelength range:

$151.4 \AA T_{5}^{-1}<\lambda_{\AA}<1514 \AA T_{5}^{-1}$,

where $T_{5}$ is colour temperature in units of $10^{5} \mathrm{~K}$, and $\lambda_{\AA}$ is wavelength in $\AA$. Hence for colour temperature of about million degrees at the beginning of the SBO, the validity range is between $15 \AA$ and $151 \AA$, and for temperature $10^{4} \mathrm{~K}$, the range is between $1514 \AA$ and $15140 \AA$.

To conclude, we suggest to compute spectral energy distribution for the SBO signal as:

$$
L_{\nu}(t)=\left\{\begin{array}{lll}
\text { Rayleign }-\operatorname{Jeans}\left(T_{\text {col }}\right) & \text {, if } & h \nu / 3 k T_{\text {col }}<0.3 \\
\text { Eq. } 2 & \text { if } \quad 0.3<h \nu / 3 k T_{\text {col }}<3 .
\end{array}\right.
$$



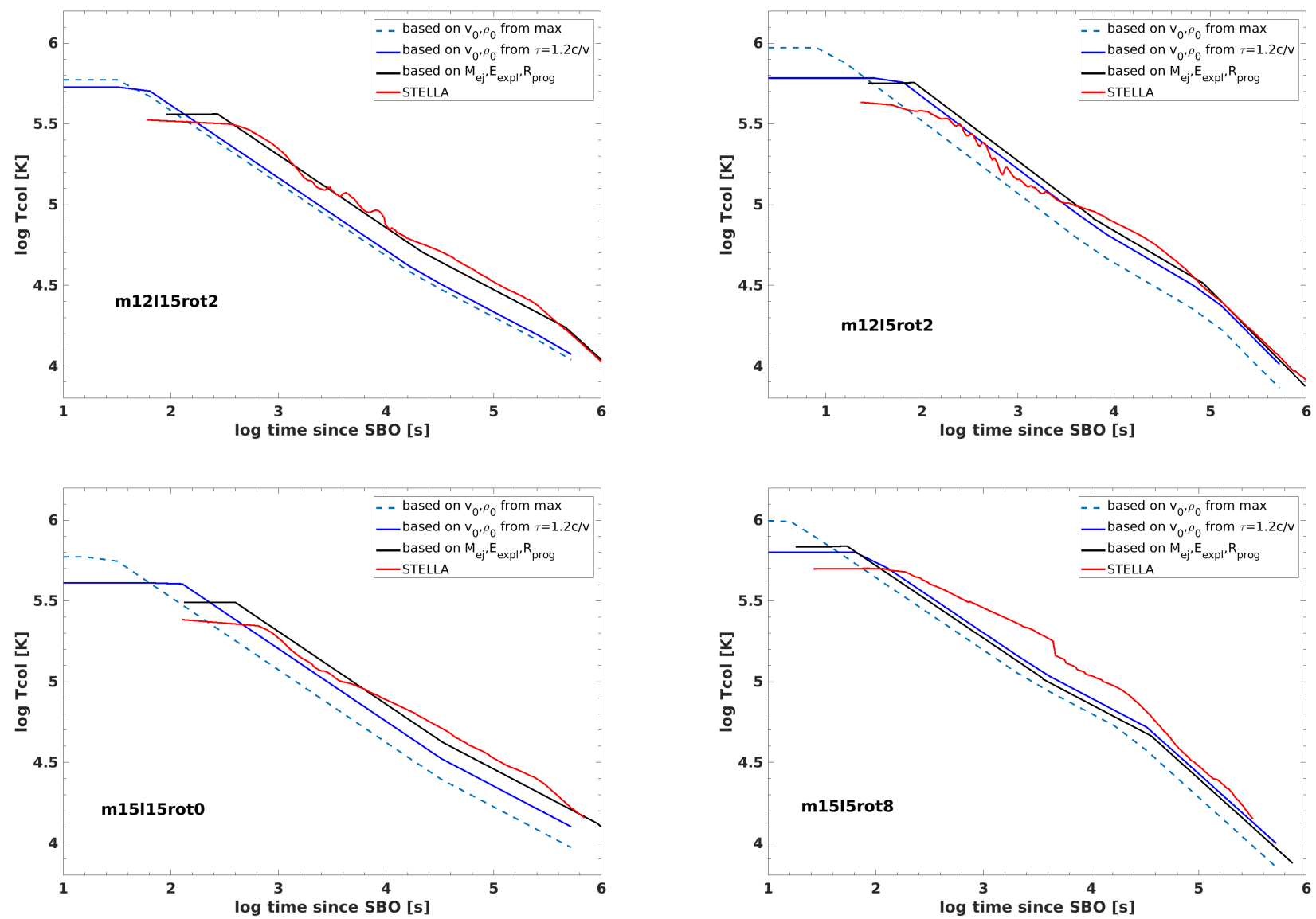

Figure 4. SBO colour temperature evolution for the red supergiant models in the study. See details in Figure 3 and in the text.

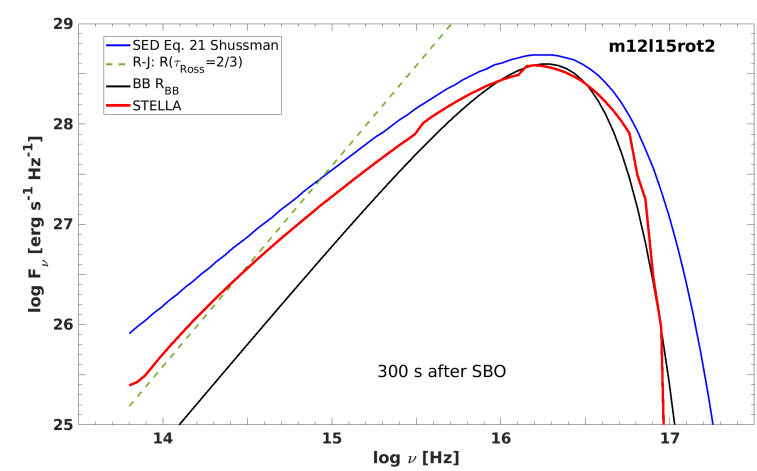

Figure 5. Spectral energy distribution for model m12l15rot2 at $300 \mathrm{~s}$ after the SBO pulse: computed with STELLA (red), calculated with analytical formulae from Shussman et al. (2016) (Eq. 19 and 21 , blue), and the black body SED at colour temperature for the given moment $T_{\mathrm{col}}=3.15 \times 10^{5} \mathrm{~K}$ (black) and RayleignJeans formulae (green). See details in the text.

\section{OTHER OBSERVATIONAL FEATURES}

\subsection{Broad-band light curves}

First of all, we present the overall light curves in different major ranges of the electromagnetic spectrum. In Fig-

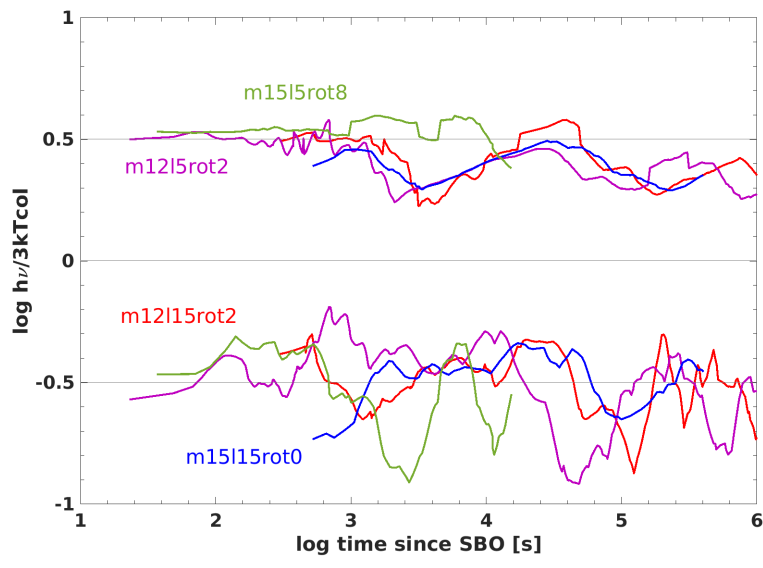

Figure 6. Evolution of spectral range (high and low frequency boundaries) in which analytic formulae from Shussman et al. (2016) are feasible.

ure 7, the magenta curve (X-ray + extreme ultraviolet) represents flux integrated between $1 \AA$ and $3250 \AA$ (corresponds to $12.4 \mathrm{keV}$ and $3.8 \mathrm{eV}$, respectively), while the red curve stands for the flux integrated between $8900 \AA$ and 50,000. "Pseudo-bol" curve (Pseudo-bolometric) stands for the flux 


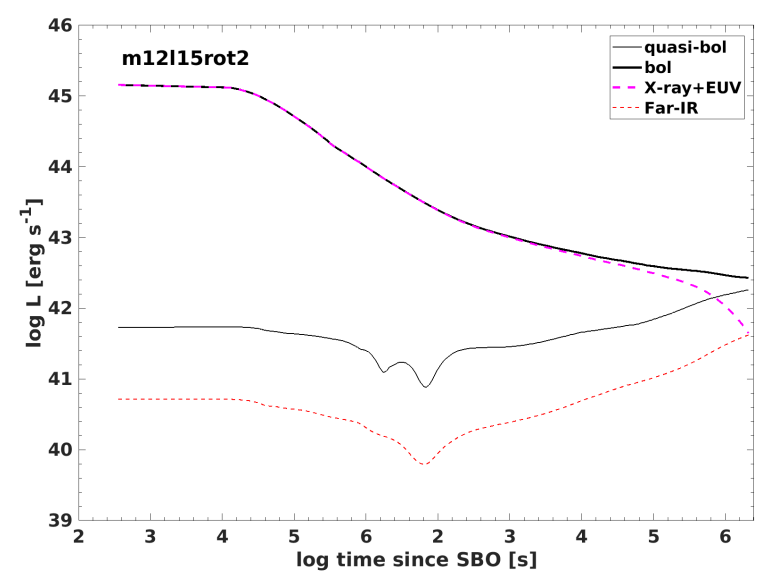

Figure 7. Light curves for the model m12115rot2: bolometric (black thick), pseudo-bolometric ( $U B V R I$, black thin), Xray+Extreme ultraviolet $(3.8 \mathrm{eV}-12.4 \mathrm{keV}$, magenta thick dashed), far infra-red $(\lambda>8900 \AA$, red thin dashed).

integrated in UBVRI spectral range, i.e. between $3250 \AA$ and $8900 \AA$. Figure 7 demonstrates that the major flux during SBO comes in the high-energy range, i.e. X-ray and ultraviolet flux overwhelmingly contributes to overall bolometric luminosity. The recently successfully launched mission SPECTRUM-RG (Merloni et al. 2012) observes the sky in the range between $0.3 \mathrm{keV}$ and $30 \mathrm{keV}$. For comparison, SBOs from normal supernovae, i.e. from exploding supergiants, have spectral maxima around $0.1 \mathrm{keV}$ at maximum light (soft X-ray), while compact Wolf-Rayet stars have SBOs in harder X-ray around $1 \mathrm{keV}$ (Nakar \& Sari 2010). Therefore, SPECTRUM-RG, particularly, eROSITA instrument will detect a moderate SBO signal from normal supernovae with a rate about a few events during a year (I. Khabibulin, R.A. Sunyaev, private communication). Ultraviolet detectors operating around $10 \mathrm{eV}$ might be more optimistic for detecting SBO from normal supernovae Sagiv et al. (2014). Figure 8 present the predicted light curves for the models in the study in the eROSITA energy window. We show two options: the solid curves are those integrated in the energy range about $0.3 \mathrm{keV}$, while dashed curves are those integrated in the window $0.5-2 \mathrm{keV}$. The later is considered as more realistic prediction due to a number of reasons, e.g. the exact dependence of the efficient telescope area on energy. On top on that, the spectrum of the SBO itself varies dramatically around $0.3-0.5 \mathrm{keV}$, and the light curve " $>0.3 \mathrm{keV}$ " and " $0.5-2 \mathrm{keV"} \mathrm{differ} \mathrm{significantly.} \mathrm{Hence,}$ in some cases (the model m1215rot2) the SBO event might be detected if consider flux above $0.3 \mathrm{keV}$ while it is at the level of the galaxy luminosity in the range $0.5-2 \mathrm{keV}$. Note that the SED peaks at higher energy for the more compact models since colour temperature is more sensitive to radius $T_{\text {col }} \sim R^{-0.46}$ rather than ejecta mass and explosion energy (see the first formula in Eq. 41). Therefore, the model m15l5rot8 (radius $268 R_{\odot}$ ) and the model m12115rt2 (345 $R_{\odot}$ ) have higher luminosity compared to other models those radii are $812 R_{\odot}$ and $1024 R_{\odot}$. We limit the plot with the luminosity $\log L=38 \mathrm{ergs}^{-1}$ because this is a typical $\mathrm{X}$-ray luminosity of a normal star-forming galaxy (Mineo

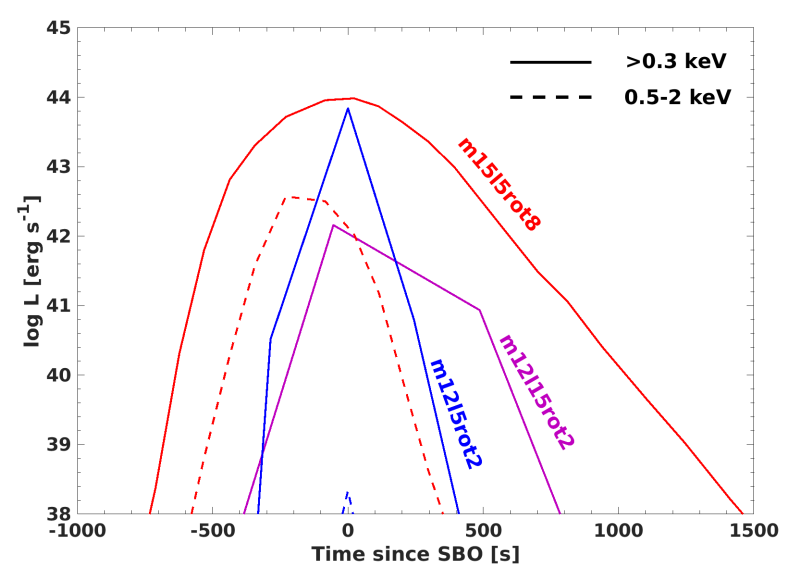

Figure 8. Predicted light curves for the models in the study as seen by eROSITA instrument in the energy range about $0.3 \mathrm{keV}$ (solid) and $0.5-2 \mathrm{keV}$ (dashed). The Y-axis limit of $10^{38} \mathrm{erg} \mathrm{s}^{-1}$ is chosen as a typical X-ray luminosity of a normal star-forming galaxy. Note that the " $0.5-2 \mathrm{keV}$ " light curve for the model m12115rot2 (supposed to be a dashed magenta curve) is below the low limit of the plot.

et al. 2012; Sazonov \& Khabibullin 2017), and events below $\log L=38 \mathrm{erg} \mathrm{s}^{-1}$ won't be detected.

As seen in Figure 7, luminosity in visible light and farinfrared range is thousand times lower than in ultraviolet, and it almost does not contribute to bolometric luminosity, although it is not negligible. Complete photometrical set is unlikely to be observed for the earlier phase, in particular, SBO pulse, since the survey telescopes usually operate with 1-2 spectral bands, and the bolometric curve could not be estimated. Even though the major flux during SBO comes from extreme ultraviolet range, some noticeable signal might be detected in visible broad bands. In Figure 9, we show broad-band light curves for the model m12115rot2. Highenergy flux is dominitating during the first 10 days, therefore, $U$ magnitude is the highest among others. $U$ stays at about $-17^{m}$ during the first $1000 \mathrm{~s}$ while $B, V, R, I$ are $-16^{m}$ for this model. At day 10, i.e. $\sim 10^{6} \mathrm{~s}, U$ peaks at $-18^{m}$ and then monotonically declines. $B, V, R$, and $I$ rebrighten after $10,000 \mathrm{~s}$, so that the major flux is radiated in these bands. According to Figure 5 and Figure 6, broad-band magnitudes in visible range might be easily computed with either the standard Rayleigh-Jeans formula for $h \nu / 3 k T_{\mathrm{col}}<0.3$, or Equation 2 (i.e. Eq. (21) from Shussman et al. 2016) for $0.3<h \nu / 3 k T_{\text {col }}<3$. The estimate of magnitudes in optical bands at earlier epoch might serve as an additional constrain to the progenitor model while analysing the entire set of observational data, like light curve, spectra, and photospheric velocity evolution.

\subsection{Photospheric velocity}

Recently, Goldberg et al. (2019) discussed that the formulae for projenitor and explosion parameters derived by Popov (1993) remain not well defined. Even being corrected for ${ }^{56} \mathrm{Ni}$ energy input and different $\mathrm{H}$-to-He ration in the envelope, Popov's formulation provides the family of models, i.e. the 


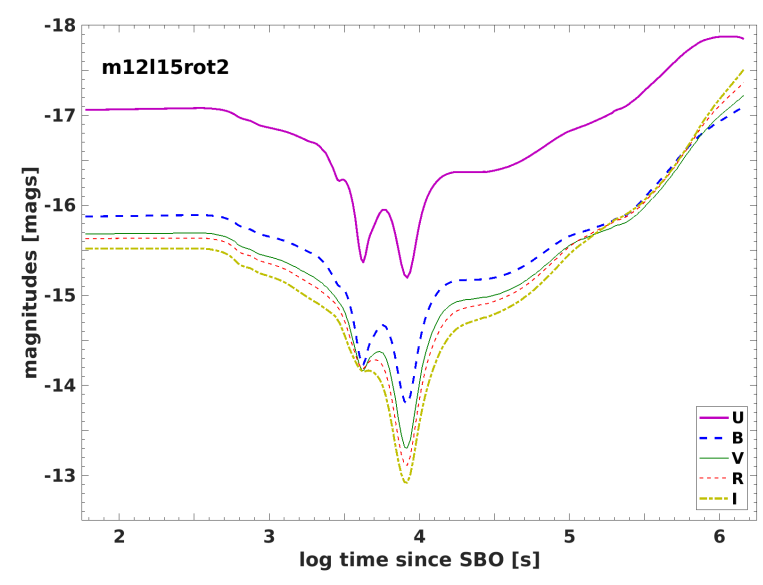

Figure 9. Broad-band light curves for the model m12115rot2.

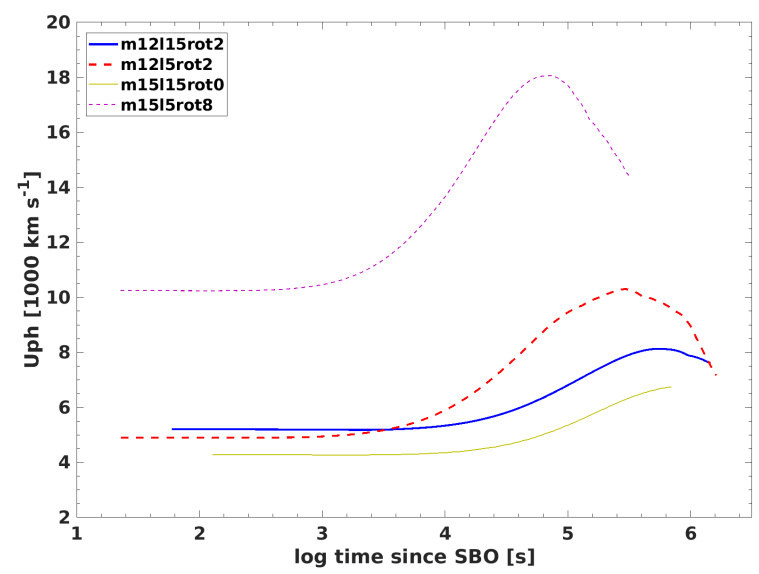

Figure 10. Photospheric velocity evolution for the models in the study during and after SBO.

combination of $M_{\mathrm{ej}}, R_{\mathrm{prog}}$ and $E_{\text {expl }}$, for a given observed SN IIP light curve. Earlier, Bersten et al. (2011) also highlighted the question about degeneracy of hydrodynamical models for a given supernova light curve. That means that the exact progenitor can not be reliably predicted based on bolometric observations such as bolometric luminosity and photospheric velocity. Therefore, additional constrains from another type of photometric observations are required. Goldberg et al. (2019) suggest to use earlier phase observations, in particular, photospheric velocity observations at day 15 , because models in the degenerated family have essentially different photospheric velocity before day 20 which strongly depends on energy of the explosion and ejecta mass (see their Fig. 21). We show photospheric velocity for the SBO phase in Figure 10. Indeed, there is a strong dependence of velocity evolution on mass and radius of the progenitor. More compact and less massive (in the sense of ejecta mass) the progenitor, the higher photospheric velocity at the maximum SBO luminosity. Note that explosion energy for all explosions in our study is the same and equal 1 foe. According to Nakar \& Sari (2010, Eq. A7) and Shussman et al. (2016,
Eq. 27a) photospheric velocity at the maximum breakout luminosity is:

$v_{0}\left[\mathrm{~km} \mathrm{~s}^{-1}\right] \approx$

$\left\{\begin{aligned} 7,000 M^{-0.43} R^{-0.26} E^{0.56} & \approx 7,000\left(\frac{E}{M R^{1 / 2}}\right)^{1 / 2}, \\ 4,500 M^{-0.44} R^{-0.49} E^{0.56} & \approx 4,500\left(\frac{E}{M R}\right)^{1 / 2},\end{aligned}\right.$

correspondingly.

In Figure 10, it is clearly seen that measuring velocity at the peak SBO signal might shed light on radius and mass of the progenitor. The matter is fully ionised at SBO, and spectra, if being recorded, are featureless. Therefore, photospheric velocity is almost unlikely to be estimated via spectral observations. However, photospheric velocity could be calculated from photometric observations via StefanBoltzmann law and a simplified assumption $v=R / t$.

Hence we suggest to use the light curve itself and colour temperature during the SBO pulse to set additional constrain for progenitor radius, ejecta mass and energy estimate.

\subsection{Dependence on global supernova parameters}

We examine the dependence of the colour temperature on explosion energy and radius based on a wider set of red supergiant models. In Figure 11, we show two models from Kozyreva et al. (2019) for which explosion energy scatters in 1 foe, between roughly 0.5 foe and 1.5 foe. This range for explosion energy is valid for the normal SNe IIP according to Pejcha \& Prieto (2015) and Müller et al. (2017) on the one hand, and also from the core-collapse explosion simulations by Ertl et al. (2016) and Sukhbold et al. (2016), on the other hand. To be precise, the model m1213 was blown up with 0.4 , 0.9 and 1.35 foe, and the model $\mathrm{m} 15 \mathrm{l} 3$ was blown up with 0.53, 1.1 and 1.53 foe. Based on Figure 11, it is clear that colour temperature weakly depends on explosion energy. We calculate the exponent for the energy dependence as:

$\alpha=\frac{\Delta T_{\mathrm{col}} / T_{\mathrm{col}}}{\Delta E / E}$

using 3 epochs: day $6,7,8$ and day 4, 5, 6 for the models $\mathrm{m} 12 \mathrm{l} 3$ and $\mathrm{m} 15 \mathrm{l} 3$, correspondingly. The averaged value for the exponent is 0.0614, which is between 0.027 (Eq. 13, Rabinak \& Waxman 2011) and 0.11 (Eq. 31, Nakar \& Sari 2010), and relatively far from -0.25 published by Shussman et al. (Eq. 41, 2016). Nevertheless, all these values are still pretty close to each other and demonstrate weak dependence of colour temperature on explosion energy.

Figure 12 shows evolution of colour temperature for a number of red supergiant models during the so-called spherical phase which precedes the recombination phase (Goldfriend et al. 2014; Faran et al. 2019). These are: $268 R_{\odot}$ and $345 R_{\odot}-\mathrm{m} 1515 \operatorname{rot} 8$ and m1215rot2 from the present study, $500 R_{\odot}$ and $631 R_{\odot}-\mathrm{m} 12 \mathrm{l} 3$ and $\mathrm{m} 15 \mathrm{l} 3$ from Kozyreva et al. (2019), $619 R_{\odot}$ - L15-pn from Limongi et al. (2000), Utrobin et al. (2017), $624 R_{\odot}-12 M_{\odot}$-model (unpublished), $812 R_{\odot}$ - m12115rot2 from the present study, $958 R_{\odot}-$ hs15.2 from Ertl et al. (2016), Sukhbold et al. (2016), $1024 R_{\odot}-$ m15115rot0 from the present study. The average ejecta mass is around $10 M_{\odot}$ and the average explosion energy is around 1 foe. Radius of the progenitors spread over the large range 


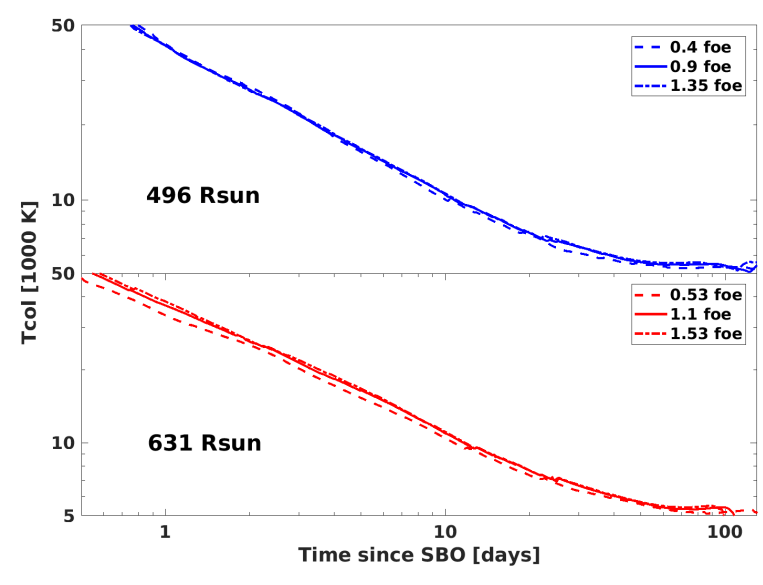

Figure 11. Colour temperature for the models m1213 and m1513 from (Kozyreva et al. 2019) for different explosion energy.

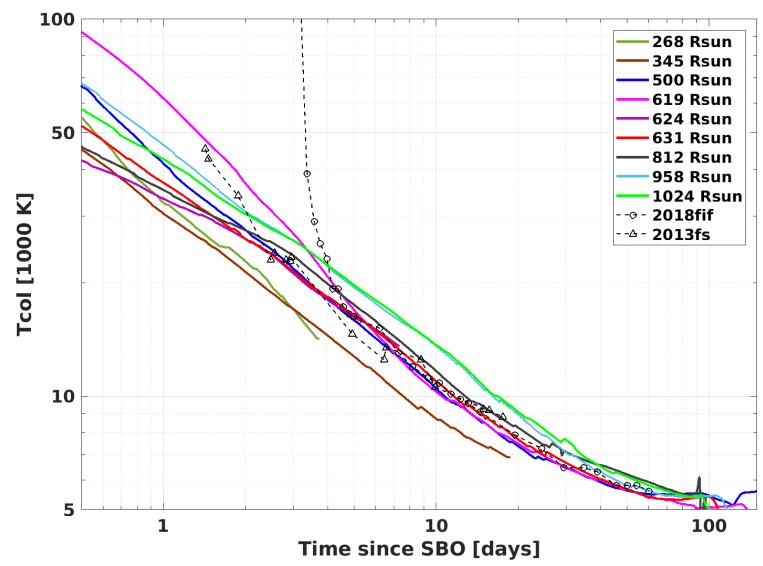

Figure 12. Colour temperature for the set of red supergiant models with different radius. Two observed supernovae are superposed with circles and triangles Soumagnac et al. (2019).

between $268 R_{\odot}$ and $1024 R_{\odot}$, and approximately cover the possible range of radii of red supergiants which explode as SNeIIP (Levesque et al. 2005, 2006; Smartt et al. 2009). Note that $T_{\text {col }} \sim M_{\mathrm{ej}}^{-\beta}$ where $\beta=0.11$ (Nakar \& Sari 2010) or $\beta=0.13$ (Shussman et al. 2016). Taking into account that dependence on mass and energy is weak, we calculate the exponent for radius dependence in a manner similar to the exponent evaluation for energy dependence. We found that $T_{\text {col }} \sim R_{\text {prog }}^{-\gamma}$ where $\gamma=0.386$ which is in very good agreement with $\gamma=0.38$ (Nakar \& Sari 2010) and sufficient agreement with $\gamma=0.46$ (Shussman et al. 2016), while $\gamma=0.25$ in studies by Rabinak \& Waxman (2011) and Sapir \& Waxman (2017). We conclude that colour temperature sets a good constrain to the progenitor radius (see also Morozova et al. 2016). However, the accuracy might not be sufficient to differenciate between progenitors at different initial masses and metallicities (Rubin \& Gal-Yam 2017). Nevertheless, this is related to a different topic of uncertainty of stellar evolution simulations (see e.g., Georgy et al. 2013; Jones et al. 2015).

\section{CONCLUSIONS}

In the present study, we run four red supergiant models from Shussman et al. (2016) with the multi-group radiation hydrodynamics code STELLA. We compare bolometric light curves and colour temperature evolution for shock breakout and relaxation after the SBO peak to the calibrated relations published in Shussman et al. (2016). We found out that analytic formulae describe reasonably well $L_{\mathrm{bol}}$ and $T_{\text {col }}$ evolution during the first day after the shock breakout, however, overestimate the absolute peak values within a factor of 3. We conclude that the analytic formulae for SBO pulse provide a fast way to estimate the possible SBO light curve and colour temperature for a given exploded progenitor, and vice versa, supernova parameters.

We show that there is good agreement between analytically and numerically computed spectral energy distribution. We provide a frequency range (Figure 6) in which analytic prescription might be reliably used Shussman et al. (2016, Equations (19) and (21)).

We show that dependence of colour temperature during the spherical phase on energy is in sufficient agreement with Rabinak \& Waxman (2011) and Nakar \& Sari (2010), but deviates from Shussman et al. (2016). The dependence of colour temperature on progenitor radius is in very good agreement with all three studes. We suggest to use colour temperature observations prior to recombination phase to set a constrain on the supernova parameters which is additional to usual estimates from the late time observations.

We present the prediction for SBO signal from the red supergiants which might be detected by the recently launched SPECTRUM-RG/eROSITA X-ray observatory.

\section{ACKNOWLEDGMENTS}

AK and EN are supported by ERC grant No. 279368 "The Gamma Ray Burst - Supernova Connection and shock breakout physics" and partially by the I-Core center of excellence of the CHE-ISF. AK is also funded by the Alexander von Humboldt Foundation. SB and PB are sponsored by grant RSF 18-12-00522. The STELLA simulations were carried out on the DIRAC Complexity system, operated by the University of Leicester IT Services, which forms part of the STFC DiRAC HPC Facility (www.dirac.ac.uk). AK thanks Ildar Khabibullin and Adam Rubin for useful and fruitful discussions.

\section{REFERENCES}

Arcavi I., et al., 2017, ApJ, 837, L2

Badjin D. A., Glazyrin S. I., Manukovskiy K. V., Blinnikov S. I., 2016, MNRAS, 459, 2188

Bersten M. C., Benvenuto O., Hamuy M., 2011, ApJ, 729, 61

Blinnikov S. I., Tolstov A. G., 2011, Astronomy Letters, 37, 194

Blinnikov S. I., Eastman R., Bartunov O. S., Popolitov V. A., Woosley S. E., 1998, ApJ, 496, 454

Blinnikov S. I., Röpke F. K., Sorokina E. I., Gieseler M., Reinecke M., Travaglio C., Hillebrandt W., Stritzinger M., 2006, A\&A, 453,229

Dessart L., Hillier D. J., 2019, A\&A, 625, A9

Dessart L., John Hillier D., Audit E., 2017, A\&A, 605, A83

Ensman L., Burrows A., 1992, ApJ, 393, 742 
Ertl T., Janka H. T., Woosley S. E., Sukhbold T., Ugliano M., 2016, ApJ, 818, 124

Faran T., Goldfriend T., Nakar E., Sari R., 2019, ApJ, 879, 20

Georgy C., Ekström S., Granada A., Meynet G., Mowlavi N., Eggenberger P., Maeder A., 2013, A\&A, 553, A24

Gezari S., et al., 2008, ApJ, 683, L131

Goldberg J. A., Bildsten L., Paxton B., 2019, ApJ, 879, 3

Goldfriend T., Nakar E., Sari R., 2014, arXiv e-prints, p. arXiv:1404.6313

Jones S., Hirschi R., Pignatari M., Heger A., Georgy C., Nishimura N., Fryer C., Herwig F., 2015, MNRAS, 447, 3115

Katz B., Sapir N., Waxman E., 2012, ApJ, 747, 147

Kozyreva A., Nakar E., Waldman R., 2019, MNRAS, 483, 1211

Levesque E. M., Massey P., Olsen K. A. G., Plez B., Josselin E., Maeder A., Meynet G., 2005, ApJ, 628, 973

Levesque E. M., Massey P., Olsen K. A. G., Plez B., Meynet G., Maeder A., 2006, ApJ, 645, 1102

Limongi M., Straniero O., Chieffi A., 2000, ApJS, 129, 625

Litvinova I. Y., Nadezhin D. K., 1985, Soviet Astronomy Letters, 11,145

Lovegrove E., Woosley S. E., Zhang W., 2017, ApJ, 845, 103

Merloni A., et al., 2012, arXiv e-prints,

Mineo S., Gilfanov M., Sunyaev R., 2012, MNRAS, 426, 1870

Modjaz M., et al., 2009, ApJ, 702, 226

Moriya T. J., 2013, PhD thesis, Department of Astronomy, Graduate School of Science University of Tokyo, $206 \mathrm{pp}$.

Morozova V., Piro A. L., Renzo M., Ott C. D., 2016, ApJ, 829, 109

Müller T., Prieto J. L., Pejcha O., Clocchiatti A., 2017, ApJ, 841, 127

Nakar E., Sari R., 2010, ApJ, 725, 904

Paxton B., Bildsten L., Dotter A., Herwig F., Lesaffre P., Timmes F., 2011, ApJS, 192, 3

Paxton B., et al., 2013, ApJS, 208, 4

Paxton B., et al., 2015, ApJS, 220, 15

Paxton B., et al., 2018, ApJS, 234, 34

Pejcha O., Prieto J. L., 2015, ApJ, 806, 225

Popov D. V., 1993, ApJ, 414, 712

Rabinak I., Waxman E., 2011, ApJ, 728, 63

Rubin A., Gal-Yam A., 2017, ApJ, 848, 8

Sagiv I., et al., 2014, AJ, 147, 79

Sapir N., Waxman E., 2017, ApJ, 838, 130

Sapir N., Katz B., Waxman E., 2011, ApJ, 742, 36

Sazonov S., Khabibullin I., 2017, MNRAS, 466, 1019

Schawinski K., et al., 2008, Science, 321, 223

Shussman T., Waldman R., Nakar E., 2016, preprint, (arXiv: 1610.05323)

Smartt S. J., 2009, ARA\&A, 47, 63

Smartt S. J., Eldridge J. J., Crockett R. M., Maund J. R., 2009, MNRAS, 395, 1409

Sobolev V. V., 1985, Moscow Izdatel Nauka, p. 504 pp.

Soderberg A. M., et al., 2008, Nature, 453, 469

Soumagnac M. T., et al., 2019, arXiv e-prints, p. arXiv:1907.11252

Sukhbold T., Ertl T., Woosley S. E., Brown J. M., Janka H.-T., 2016, ApJ, 821, 38

Tolstov A. G., Blinnikov S. I., Nadyozhin D. K., 2013, MNRAS, 429,3181

Tominaga N., Morokuma T., Blinnikov S. I., Baklanov P., Sorokina E. I., Nomoto K., 2011, ApJS, 193, 20

Utrobin V. P., Wongwathanarat A., Janka H.-T., Müller E., 2017, ApJ, 846, 37

\section{APPENDIX A: ANALYTICAL FORMULAE FROM SHUSSMAN ET AL. 2016}

\section{A1 Bolometric light curve and observed temperature as functions of the breakout properties (Section 5.1, Shussman et al., 2016)}

In Equation 33, 35, and 42 below we use velocity of the SBO shell prior to SBO $v \equiv v_{0,5}$ in units $5,000 \mathrm{~km} \mathrm{~s}^{-1}$, density $\rho \equiv \rho_{0,-9}$ in units $10^{-9} \mathrm{~g} \mathrm{~cm}^{-3}$, and radius of the progenitor prior to SBO $R \equiv R_{500}$ in units $500 R_{\odot}$.

Equation 33:

$L_{\text {obs }}(t)\left[\mathrm{erg} \mathrm{s}^{-1}\right] \simeq$

$\left\{\begin{array}{lllllc}1.6 \times 10^{45} & v^{3} & \rho & R^{2} & & t<t_{0} \\ 3.2 \times 10^{43} & v^{0.33} & \rho^{-0.33} & R^{-1.69} & t_{h r}^{-4 / 3} & t_{0} \ll t \ll t_{s} \\ 3.3 \times 10^{42} & v^{1.31} & \rho^{-0.33} & R^{0.71} & t_{\text {day }}^{-0.35} & t_{s} \ll t<t_{\text {rec }}\end{array}\right.$

Equation 35:

$T_{\mathrm{obs}}(t)[\mathrm{K}] \simeq$

$\left\{\begin{array}{lllllc}4.2 \times 10^{5} & v^{0.76} & \rho^{0.24} & & & t<t_{0} \\ 1.1 \times 10^{5} & v^{-0.13} & \rho^{-0.21} & R^{-0.1} & t_{h r}^{-0.45} & t_{0} \leq t<t_{s} \\ 3.3 \times 10^{4} & v^{-0.03} & \rho^{-0.2} & R^{-0.2} & t_{\text {day }}^{-0.35} & t_{s} \leq t<t_{c} \\ 4.1 \times 10^{4} & v^{-0.55} & \rho^{0.18} & R^{0.06} & t_{\text {day }}^{-0.6} & t_{c} \leq t<t_{\text {rec }}\end{array}\right.$

Here, $t_{0}, t_{s}, t_{c}$, and $t_{r e c}$ are so-called transition times. $t_{0}$ marks the end of maximum SBO luminosity phase and the beginning of planar phase, $t_{s}$ is the end of planar phase and the beginning of spherical phase, and $t_{r e c}$ is the end of spherical phase and the beginning of recombination phase. $t_{c}$ is so-called "colour" transition time, when thermalization point reaches the breakout shell and as a consequence colour temperature undergoes a break in its temporal evolution during spherical phase (Section 3.2, Shussman et al. 2016). We list the expressions for the transition times below in Equations $36 \mathrm{a}, 36 \mathrm{~b}, 36 \mathrm{c}$, and $36 \mathrm{~d}$ :

$$
\begin{array}{llll}
t_{0}=190 \mathrm{~s} & v^{-2} & \rho^{-1} & R^{-0.23} \\
t_{s}=3.2 \mathrm{hr} & v^{-1} & & R \\
t_{c}=2.5 \text { days } & v^{-2.07} & \rho^{0.08} & R^{1.06} \\
t_{\text {rec }}=17 \text { days } & v^{-0.92} & \rho^{-0.31} & R^{0.1}
\end{array}
$$

\section{A2 Bolometric light curve and observed temperature as functions of global $\mathrm{SN}$ properties (Section 5.2)}

In the equations below we use radius of the progenitor prior to SBO $R \equiv R_{500}$ in units $500 R_{\odot}$, ejecta mass $M \equiv M_{15}$ in units $15 M_{\odot}$, energy of the explosion $E \equiv E_{51}$ in units $10^{51} \mathrm{erg}$.

Equation 39:

$L_{\text {obs }}(t)\left[\operatorname{erg~s}^{-1}\right] \simeq$

$\left\{\begin{array}{llllll}1.8 \times 10^{45} & M^{-0.65} & R^{-0.11} & E^{1.37} & & t \ll t_{0} \\ 2.7 \times 10^{43} & M^{-0.34} & R^{1.74} & E^{0.29} & t_{\mathrm{hr}}^{-4 / 3} & t_{0} \ll t \ll t_{s} \\ 1.6 \times 10^{42} & M^{-0.78} & R^{0.28} & E^{0.84} & t_{\text {day }}^{-0.35} & t_{s} \ll t<t_{r e c}\end{array}\right.$

Equation 41:

$T_{\text {obs }}(t) \simeq$ 


$\left\{\begin{array}{rlllll}4.3 \times 10^{5} & M^{-0.17} & R^{-0.52} & E^{0.35} & & t<t_{0} \\ 1 \times 10^{5} & M^{-0.07} & R^{0.1} & E^{-0.01} & t_{\mathrm{hr}}^{-0.45} & t_{0} \leq t<t_{s} \\ 3 \times 10^{4} & M^{-0.11} & R^{-0.04} & E^{0.04} & t_{\text {day }}^{-0.35} & t_{s} \leq t<t_{c} \\ 4.1 \times 10^{4} & M^{0.13} & R^{0.46} & E^{-0.25} & t_{\text {day }}^{-0.6} & t_{c} \leq t<t_{r e c}\end{array}\right.$

Equations 42a, 42b, 42c, 42d:

$\begin{array}{llll}t_{0}=155 \mathrm{~s} & M^{0.23} & R^{1.39} & E^{-0.81} \\ t_{s}=3.6 \mathrm{hr} & M^{0.44} & R^{1.49} & E^{-0.56} \\ t_{c}=3.2 \text { days } & M^{0.97} & R^{2.02} & E^{-1.19} \\ t_{\text {rec }}=16.6 \text { days } & M^{0.22} & R^{0.76} & E^{-0.43}\end{array}$

This paper has been typeset from a $\mathrm{T}_{\mathrm{E}} \mathrm{X} / \mathrm{LAT} \mathrm{E} \mathrm{X}$ file prepared by the author. 